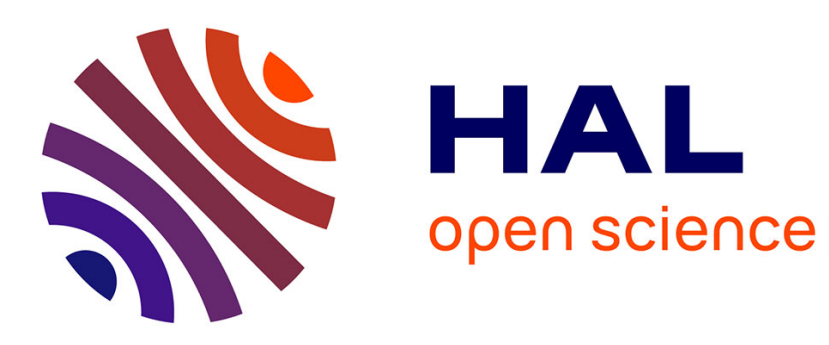

\title{
BIM-Agile Practices Experiments in Architectural Design
}

Henri-Jean Gless, Damien Hanser, Gilles Halin

\section{To cite this version:}

Henri-Jean Gless, Damien Hanser, Gilles Halin. BIM-Agile Practices Experiments in Architectural Design: Elicitation of Architectural Intentions and Refinement of Design Tasks. CDVE 2017, Cooperative Design, Visualization, and Engineering, Sep 2017, Mallorca, Spain. pp.135-142, 10.1007/9783-319-66805-5_17. hal-02868729

\section{HAL Id: hal-02868729 \\ https://hal.science/hal-02868729}

Submitted on 18 Jun 2020

HAL is a multi-disciplinary open access archive for the deposit and dissemination of scientific research documents, whether they are published or not. The documents may come from teaching and research institutions in France or abroad, or from public or private research centers.
L'archive ouverte pluridisciplinaire HAL, est destinée au dépôt et à la diffusion de documents scientifiques de niveau recherche, publiés ou non, émanant des établissements d'enseignement et de recherche français ou étrangers, des laboratoires publics ou privés. 


\title{
BIM-Agile Practices Experiments in Architectural Design
}

\section{Elicitation of Architectural Intentions and Refinement of Design Tasks}

\author{
Henri-Jean Gless, Damien Hanser, and Gilles Halin \\ MAP-CRAI, 2 rue Bastien Lepage, 54000 Nancy, France
}

\begin{abstract}
The digital transition is changing the way architectural firms are making design. The BIM technology, which tends to become mandatory for legal and competitive reasons is both convincing because of its parametric and global modeling sides and frightening because of changes caused by the arrival of new digital tools. Indeed, our basic postulate is that the emergence of new digital tools must necessarily be followed by the emergence of new practices and new project management in design stage.

This research focuses on innovative project management methods and collaborative practices allowing to facilitate the integration of new digital tools in order to create innovative practices and methods adapted to computer-assisted and collaborative architectural design. We take inspiration from agile methods and practices born in the software engineering world in the 1990s. Agile methods are innovative project management methods that focus mainly on a better reactivity. We have thus identified that a better reactivity is corroborated to a better collaboration around the understanding and repartition of design tasks.

Thus, we focus in particular in this paper on elicitation of architectural intentions and refinement of design tasks in collaborative groups of students working on a BIM project. For this purpose, we have set up a collaborative matrix that students fill up by explaining together their architectural wills and intentions for this project exercise. Naturally follows a defining "tasks to be done" process, which we will detail in this paper.
\end{abstract}

Keywords: BIM - Agile methods - Agile practices - Collaboration Architectural design : Project management - Collaborative and digital uses Collaborative and digital practices

\section{Introduction}

Our research takes place in the French architectural design field where the digital transition still has not succeeded to implement BIM technology. Nowadays, on the field, we notice that this transition is only seen through a technological approach. We 
make the assumption that this could be a major reason explaining why the transition is still at an early stage of the BIM evolution.

We propose in this article to adopt a more human-centered approach in order to make evolve project management methods relying on BIM technology. Current methods need to be improved to complete the digital transition, and in this research, we seek to evaluate if methods used in software engineering, like so-called agile project management methods can be applied to the architectural field. These methods have been created to meet the needs of a greater responsiveness and customer involvement, and for being applied on a non-linear and iterative design process, as such architectural design [1]. Links between BIM technology and agility are numerous: such as adaptability to change, the will to reduce information redundancies, or to improve communication between actors [2, 3].

The experiments we are conducting propose to apply agile design practices in architectural school study projects, in order to help them correctly identify design tasks, their perimeters as also their complexity. This experiment protocol consists of three steps: the students translate collaboratively their architectural intentions, then identify the tasks that they should do, and finally estimate their complexity and duration. These steps are done using agile practices based on a tool for design assistance called "Conceptual matrix" and an agile practice called "Planning Poker". Expected results are improvements in adaptability, reduction wastes and communication.

\section{Context of AEC in France}

The world of architecture, engineering and construction (AEC) in France, more particularly in the field of architectural design, is currently going through an important period of changes; digital field as well as collaborative practices are in a phase of transition and adaptation while the BIM technology becomes a regulatory demand in public construction. However, there is some inertia from architects towards Building Information Modeling. This trend is based on the French architecture firms' size. Most of them are small $(90 \%$ of it have 9 or fewer employees, and $75 \%$ have 4 or fewer employees) [4], but also in the current socio-economic context which encourages low investment in the medium and long term.

In the construction context, proven working methods, such as LEAN Management for production activities or agile methods developed in the software engineering field, tend to apply to the construction field. We can mention LEAN Construction [5], which particularly targets the management of working site, or methods which bet on humans and lead to a reduction of waste and accidents [6]. It aims to make the actors responsible and to ensure that they take care of each other, while trying to anticipate everyone's needs. BIM technology and these innovative project management methods, and especially agile methods [2,3] share common values such as the application of better communication and a significant reduction in the "work to be redone" [3].

The experiments we propose are part of a research focusing on agile practices identification, their implementation in BIM oriented experiments for measuring their benefits within architectural student's projects on the one hand, and on the other in architectural firms. 
Our analysis is that BIM implementation issue cannot be resumed by a simple technological problem, it is crucial to study the situation in the light of a global digital transition of the architecture firms through a collaborative practices approach.

Indeed, architecture students and architectural firms we meet are becoming more and more informed and aware of the concept of BIM, but we noticed that the task definition remains a difficult activity to perform in the design process because it often depends on other tasks performed by other actors, or simply difficult to quantify.

\section{Agile Practices Experiments in Architectural Design}

\subsection{Toward an Agile Architectural Conception}

Agile methods are innovative project management methods that have emerged in the software engineering field in the 1990s. Wanting to be more pragmatic than traditional methods, they involve more the customer in the production chain by realizing numerous feedbacks and demonstrations in order to correctly identify his needs. They also follow three other fundamental values which are: collaborating team, working production, and responding to change. In addition to these four values, we found twelve agile principles which are by example a frequent work delivery, encourage face-to-face conversations, or making emerge self-organizing teams.

We can also add that these methods are based on an iterative, incremental and evolutionary productive cycle. On regular cycles, we develop a functional model improved by incremental functions. This model regularly evolves with the customer feedbacks. We then can see the links which are forged between the BIM technology and agile methods. The true nature of BIM technology is to evolve by design cycles, and its parametric side allows it to be responsive to change.

We have thus oriented our work towards methods allowing the different actors of a project to better identify the definition of a task in its whole complexity. We were inspired by the Suh's matrices [7] and created a table called "Conceptual Matrix" adapted to an agile architectural design.

Conceptual matrix is a tool that allows to "write down" the designers" architectural intentions. This matrix makes it possible to collaboratively express the needs or intentions of a project in order to prepare the realization of BIM tasks. These tasks will be first "refined" by the use of another agile practice: the "planning poker".

\subsection{Elicitation Process: Writing Down Architectural Intentions}

Elicitation is the act of formalizing an idea, a concept or an intention to be understood by its interlocutors. Or, in a collaborative context, the main goal is to be on the same page as its coworkers.

For this purpose, we have designed a conceptual matrix that project actors fill up together to confront their ideas and their understandings of architectural subjects.

As we seen in Fig. 1, conceptual matrix is an online spreadsheet where we find in rows the inputs (the intrants): the programmatic elements of a project, the needs of the 


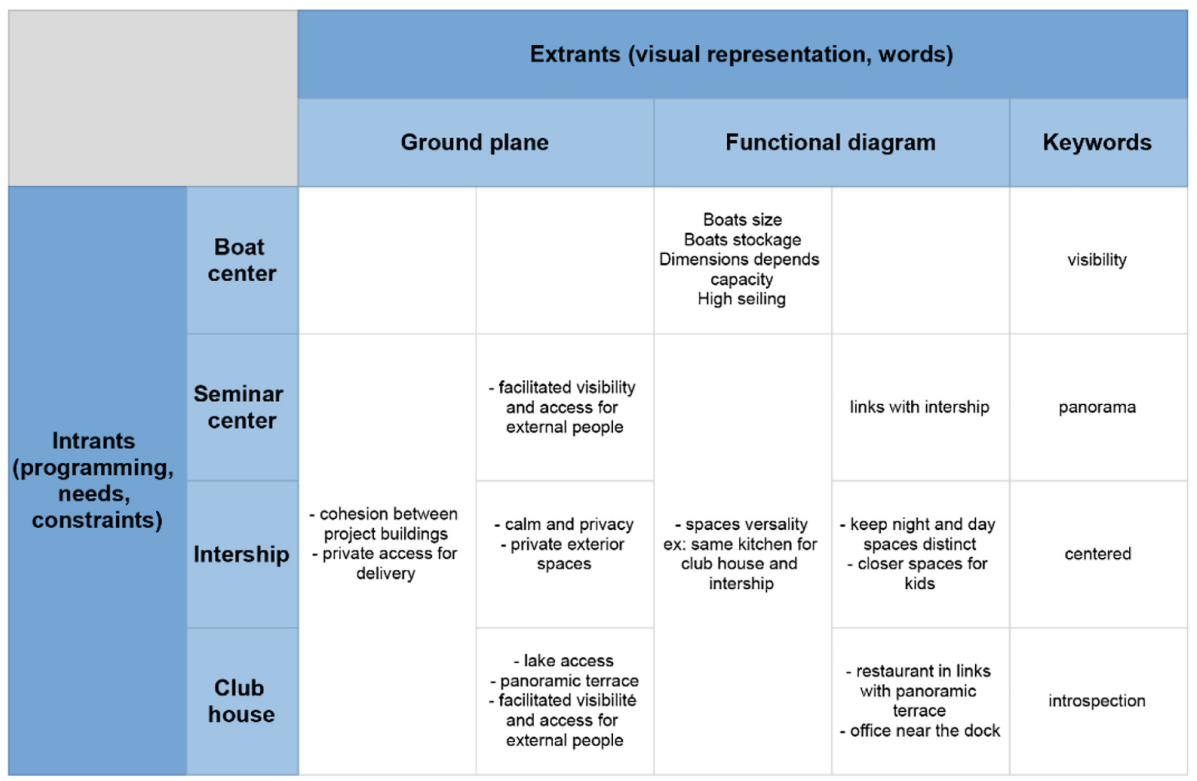

Fig. 1. Example of a conceptual matrix filled up by one student group

project manager or his constraints; and in columns the outputs (the extrants): the geometric or semantic translation of the inputs.

The conceptual matrix allows the different actors of a project to formalize conceptual elements by keywords and sentences to compare each other ideas. The main goal is not to fill all the spreadsheet cases, but to give actors a way to collaboratively determinate concepts and expectations by dialogue and debate. The matrix is a tool which valid intentions explorations.

Once achieved, this conceptual matrix makes it possible to realize a list of tasks to be executed, which we will define and specify with a planning poker.

\subsection{Refinement Process: Perimeter Identification and Duration Estimation of Tasks}

We have formalized the definition of a task according to the following characteristics:

- Title: how we name a task

- Complexity: allows a debate to define what constitutes the task

- Duration: in minutes or hour with a deadline

- Attribution: who will be in charge of this task

While title is defined after realization of the conceptual matrix, the other characteristics are defined during a planning poker. The planning poker is set of 13 cards with facial values from 1 to 100 and following an exponential distribution $(1,2,3,5,8,13$, 20, 40 and 100). 
Each turn corresponds to the definition of a task $\mathrm{n}$. Thus, for the definition of the complexity of the task $\mathrm{n}$, each player chooses a card of his game, poses face down in front of him, then return them at the same time. The objectives are to encourage negotiation between participants, avoid biased opinions following the first speaker, and allow all actors to speak (the "I do not know" card does not exist). When the discussion is over, participants choose a compromise value before doing the same process for the duration estimation, then move with the next task.

In Fig. 2, time estimations of each group 1 student for each task have been reported under the "Student x" label. "Estimation" bar is the final result of the group estimation after debating. "Real time" is the time measured at the end of the task. These last two values are reported in the Fig. 3.

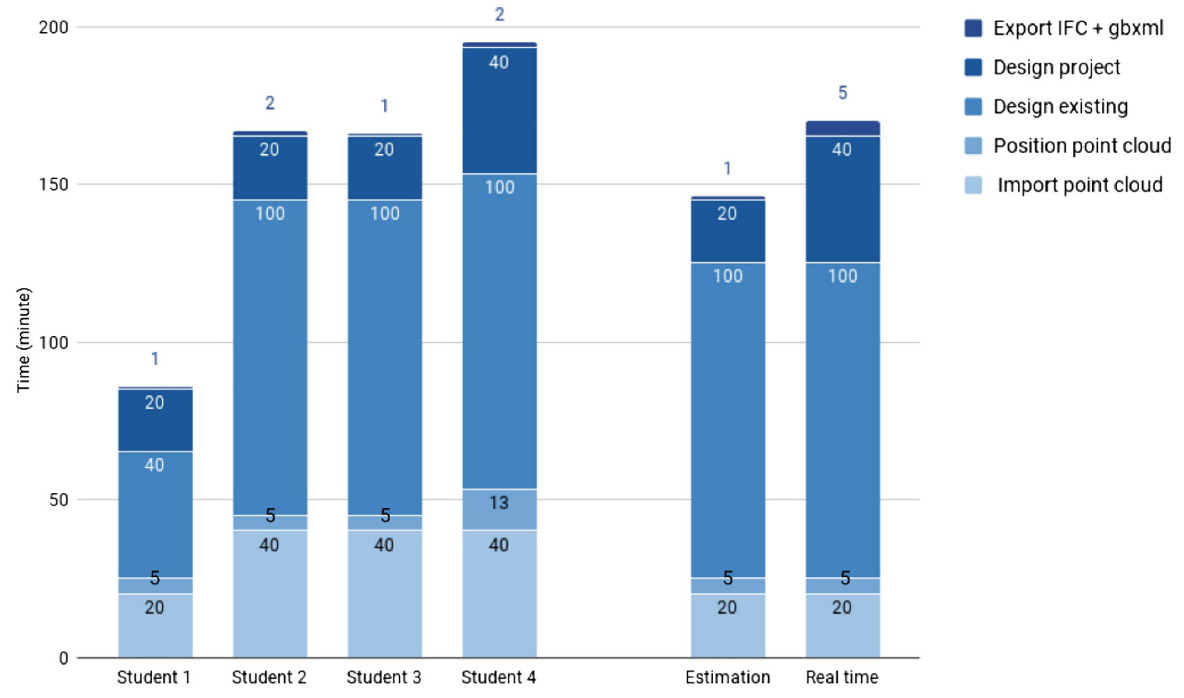

Fig. 2. Students estimations for a BIM exercise with planning poker

Even if their estimates of time or complexity are mistaken, they allow the actors of a project to start the negotiations, to find who does what, who has the best skills to carry out the task, and bring them closer to the optimal effective duration. Indeed, necessary exchanges and debates permitted to highlighting the different skills and expectations of each actor. We then notice that high or low estimations of time for realize a task show either a lack of knowledge about the subject complexity, or on the contrary a high appreciation due to an expert view. Ensuing exchanges permit all the actors to understand the overall scope of the task to be accomplished. 


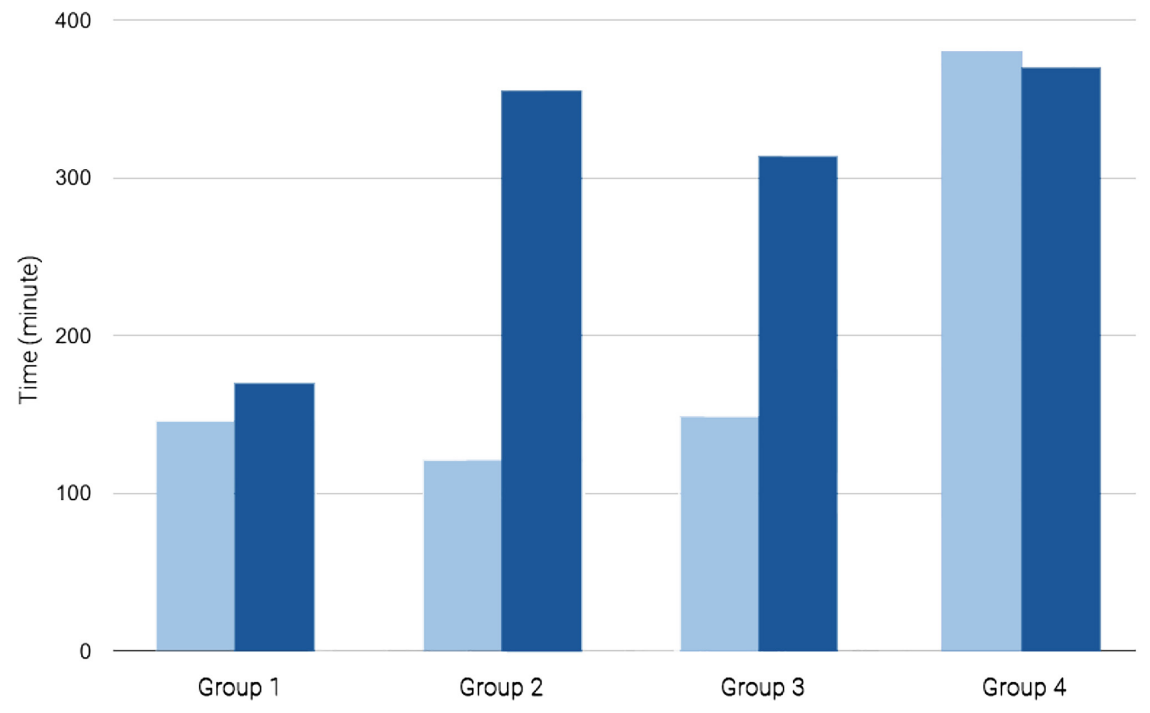

Fig. 3. Students estimations and real durations of the BIM exercise

\subsection{Protocol and First Experiments}

\section{Protocol}

During a short BIM collaborative school exercise, Master 2 architecture students have had to use these processes to properly express their architectural desires and intentions. The groups were composed of architects students, building engineers students, and general engineers students.

We followed this protocol:

- Theoretical phase: introductive course on agility, followed by a planning poker explanation.

- Practical phase: conceptual matrix presentation, filled collaboratively.

- Practical phase: matrix translation in tasks (titles), followed by a planning poker.

- Practical phase: project modeling, where durations are measured by students and reported in a table for analysis.

- Data gathering: students make a report of their matrices, results of planning poker and real tasks durations, with a comment on the practices.

- Data analysis: bias sort and practices improvement.

The theoretical phase allows the students to understand the challenge involved in making the project management evolve in parallel with the digital tools, and to adopt simple and effective agile methods. The practical phases enable students to apply the 
presented collaborative practices as exercises. Finally, data phases allow students to debrief their work and their matrices in order to adopt a task reference estimation for their future project; they also allow us to have students' feedbacks on the experiments, in order to improve the elicitation of intentions and the refinement of tasks process for the next school year.

Since spreadsheets matrices are online, we can collect data after the experiment. We then compare estimations in these spreadsheets with the real duration work, and sort concluding bias like bad subject comprehension, computer failure, or any unexpected event (keeping in mind that adaptability to change is nature of agile).

\section{Experiments}

The next figure shows estimations and measurements of duration tasks. Students have converted the conceptual matrix in a task table with planning poker corresponding the practical phases. Their goal was to import a point cloud from another course they followed before, position it in a BIM software, design the existing building thanks to this point cloud, design their architectural project with the help of the conceptual matrix and export the result for another course. The class was divided into four groups of four students, mixed academic origins and skills.

As we see in Fig. 3, some total estimations and real durations can be really different. Three groups estimate total work for 120-150 min, the last one 380. Final durations are between 310 and $370 \mathrm{~min}$. The gap is due to hard tasks (import point cloud and position it) that only Group4 has planned. The Group1 has not accounted in its estimation this task, and has just got the work of another group. Sort this two tasks, each group correctly estimate their design tasks with the help of the conceptual matrix and dialogues and debate resulting from.

\section{Conclusion and Perspectives}

The experiments propose a defined process for students to make collaborative projects. The exchanges allow to collaboratively filling up spreadsheets in a rational and objective way to compare and measure wills and architectural intentions. Following by a planning poker, they can estimate the duration of their work in a BIM environment by an efficient manner thanks to their own skills and experiences.

The perspectives opening to us are now twofold. First, a work on field to implement our agile-BIM practices with an architectural firm wanting to realize a BIM transition. After the translation of agile methods to architectural field, we must then confront and transmit them to professionals. Indeed, we will commit ourselves to testing these innovative practices for the agencies, having no R\&D department. Second, another pedagogical experiment during a semester duration project studio. We will be then able to confront our work with long architectural projects. Moreover, this paper shows the first two agile practices that we have experienced, which are more team-oriented. The next ones will be more customer-oriented, like a scrum-like method translated for our needs, a stand-up meeting, and a management dashboard which will be supported and fed with our matrix, providing an overall project view for the designers. 


\section{References}

1. Halin, G., Hanser, D., Bignon, J.-C.: User adaptive visualization of cooperative architectural design. Int. J. Archit. Comput. 2(1), 89-107 (2004). doi:10.1260/1478077041220188

2. Succar, B.: Building information modelling framework: a research and delivery foundation for industry stakeholders. Autom. Constr. 18(2009), 357-375 (2009). doi:10.1016/j.autcon. 2008.10.003

3. Beck, K., et al.: Manifesto for agile software development (2001). http://agilemanifesto.org

4. Numbers and maps of the architectural profession, a professional environment composed mainly of small structures, p. 48 (2015). http://www.architectes.org/sites/default/files/atoms/ files/archigraphie-light_1.pdf

5. Dupin, P.: LEAN applied to construction: how to optimize project management and reduce costs and delays in building? Eyrolles (2014). ISBN 978-2212138320

6. Womack, J., Jones, D.: Lean Thinking: Banish Waste and Create Wealth in Your Corporation, 2nd edn. Revised and Updated. Free Press (2003). ISBN 978-0743249270

7. Suh, N.: Axiomatic Design: Advances and Applications. Oxford University Press, Oxford (2001). ISBN 978-0195134667 